\title{
Short Nuss bar procedure
}

\author{
Hans Kristian Pilegaard ${ }^{1,2}$ \\ ${ }^{1}$ Department of Cardiothoracic and Vascular Surgery, Aarhus University Hospital, Skejby, Aarhus, Denmark; ${ }^{2}$ Department of Clinical Medicine, \\ Aarhus University, Aarhus, Denmark \\ Correspondence to: Hans Kristian Pilegaard. Department of Cardiothoracic and Vascular Surgery, Aarhus University Hospital, Skejby, Palle Juul- \\ Jensens Boulevard 99, DK-8200 Aarhus N, Denmark. Email: pilegaard@dadlnet.dk.
}

\begin{abstract}
The Nuss procedure is now the preferred operation for surgical correction of pectus excavatum (PE). It is a minimally invasive technique, whereby one to three curved metal bars are inserted behind the sternum in order to push it into a normal position. The bars are left in situ for three years and then removed. This procedure significantly improves quality of life and, in most cases, also improves cardiac performance. Previously, the modified Ravitch procedure was used with resection of cartilage and the use of posterior support. This article details the new modified Nuss procedure, which requires the use of shorter bars than specified by the original technique. This technique facilitates the operation as the bar may be guided manually through the chest wall and no additional stabilizing sutures are necessary.
\end{abstract}

Keywords: Pectus excavatum repair (PE repair); Nuss; short bar; minimally invasive surgery

Submitted Mar 14, 2016. Accepted for publication Jul 12, 2016.

doi: 10.21037 /acs.2016.09.06

View this article at: http://dx.doi.org/10.21037/acs.2016.09.06

\section{Introduction}

Pectus excavatum (PE) is the most common anomaly of the anterior chest wall. Correction of the PE significantly increases the quality of life for the patients and in most cases also improves their cardiac performance $(1,2)$. Since Ravitch published his first paper in 1949, a modified Ravitch approach with resection of cartilage has been the preferred operation for surgical correction of $\mathrm{PE}$ in most surgical units (3). After Nuss published his minimally invasive Nuss procedure in 1998, whereby 1-3 curved bars are inserted behind the sternum to position it anteriorly, it has changed the treatment of PE and become the most commonly used technique (4). It results in improved cosmesis regarding both shape of the anterior chest wall and the absence of any incision anterior to the sternum. The original technique prescribes a bar length from one mid axillary line to the other. The technique has since been modified with the use of shorter bars (5-7).

\section{Indication}

The Nuss procedure may be used to correct all cases of PE.
The optimal age for the operation is still under discussion. According to the findings of Nuss, there is an increased risk of recurrence if the operation occurs before puberty. Therefore, most surgeons find that the beginning of the puberty is the optimal time for correction. It may be suitable to operate on female patients at a slightly older age, when they have some degree of demarcation of the mammary fold, which facilitactes a better cosmetic placement of incisions in the sub-mammary line. The upper age limit for the procedure is not known. Patients over the age of 60 have been operated on due to symptoms with acceptable results (8).

Most patients request surgical correction of $\mathrm{PE}$ due to cosmetic improvement but more than $60 \%$ also complain of exercise intolerance and shortness of breath (9).

Preoperative examination includes clinical examination, standard blood tests and X-rays in two planes. Computed tomography (CT) imaging or echocardiography is only carried out if there are grounds to suspect intra-thoracic pathology, such as for patients with Marfan syndrome.

\section{Preparation}

The operation is performed under general anesthesia. The 


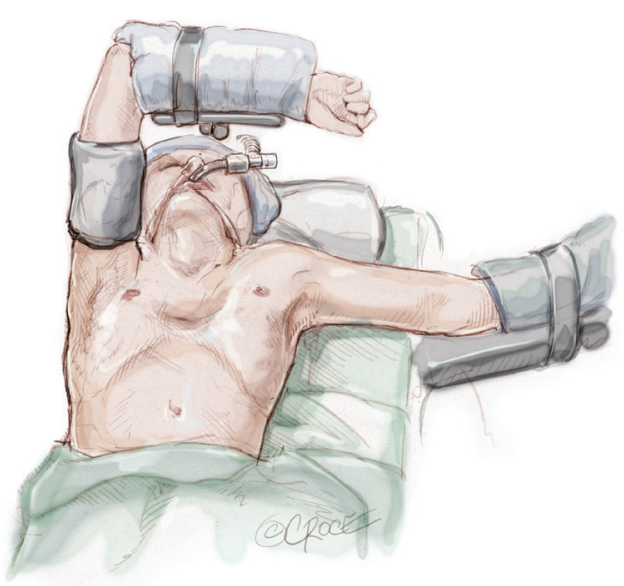

Figure 1 The position of the patient on the OR table.

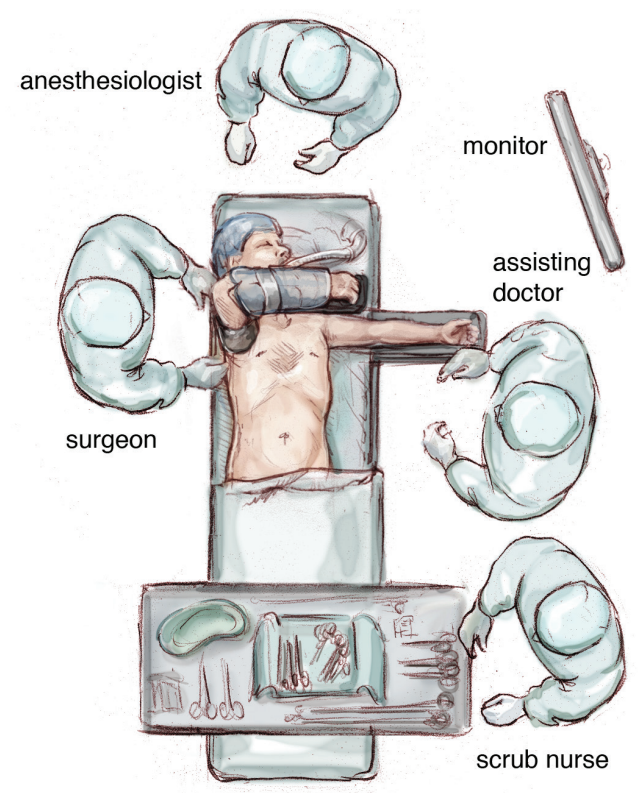

Figure 2 Shows the level of the port for the thoracoscope and the location of the OR team around the patient.

patient is equipped with a urinary catheter, an arterial line and intubated by a single-lumen tube. A double-lumen endotracheal tube is only used if there has been previous thoracic intervention or infection.

\section{Exposition}

The patient is positioned on the right side of the table in a supine position with the right arm in front of the head (Figure 1). The surgeon stands on the right side and the

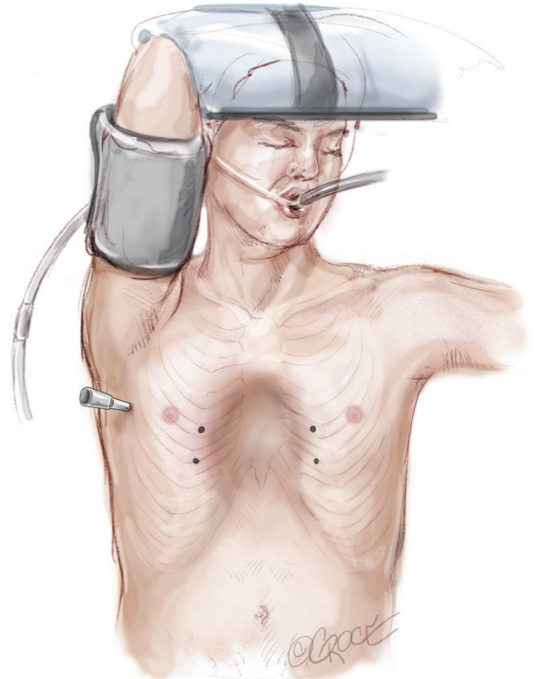

Figure 3 Marking of the points for entrance and exit of the bars through the chest wall.

assistant on left. A 5-mm port is placed through the right lateral side of the chest, at the level of the nipple (Figure 2).

\section{Operation}

The thoracoscope is inserted through the port and intrathoracic structures are visualized while the patient is in apnea. Any adhesions identified are cut with a harmonic scalpel prior to further surgery. The deepest point under the PE is then defined. This is not always the same point as seen externally. The entry and exit points for the bar through the chest wall are marked (Figure 3). The entry point should be just medial to the most superior point of the deformity. Pressing on this point will confirm its correct position if intercostal tissue movement is in line with the deepest point as visualized with the thoracoscope. Dissection under the sternum is performed under apnea and may take less than a minute.

\section{Bars}

In most cases, the bar is placed so that it supports the deepest point of the $\mathrm{PE}$ and runs straight across the chest. Sometimes the deepest point is between two intercostal spaces and therefore correction may be optimized if the bar is placed in an oblique position (Figure 4). If the PE is very long, two or three bars might be needed to optimize correction. In some cases, bars may be even placed in a 

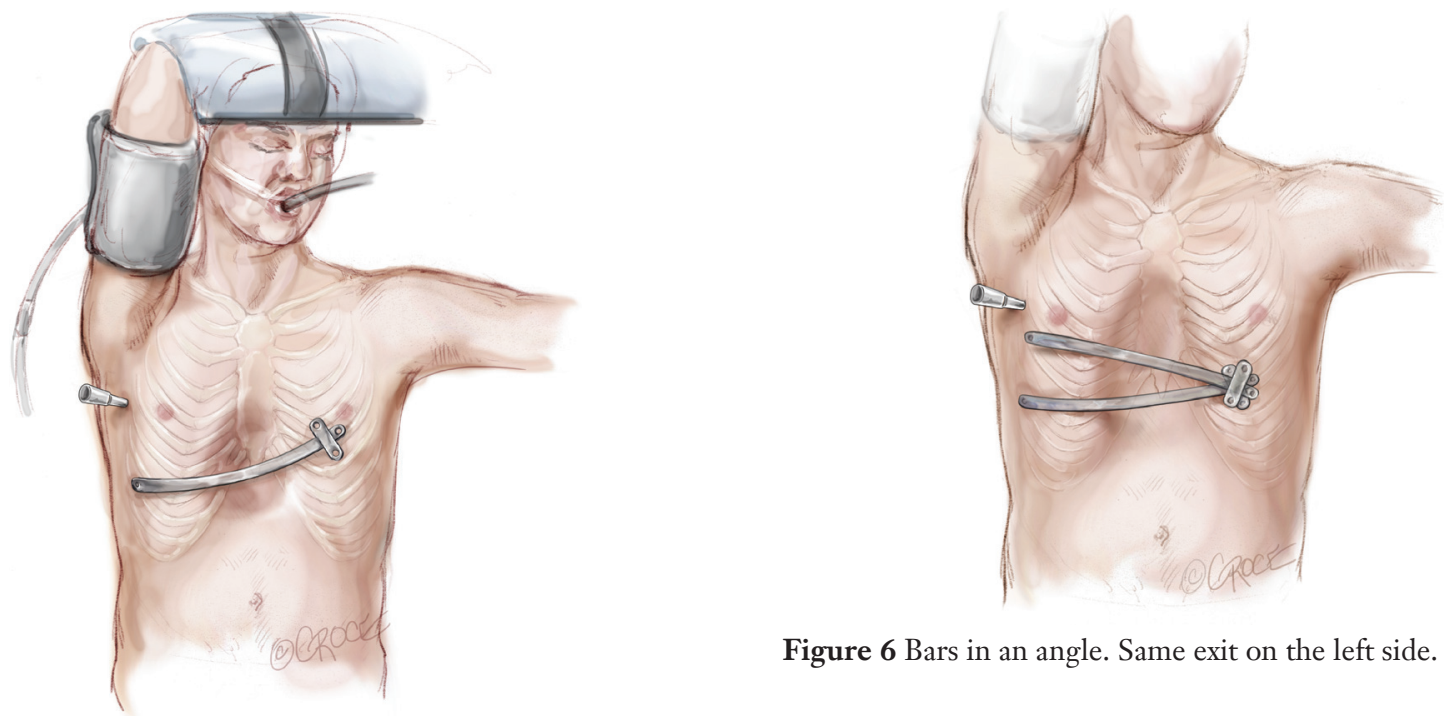

Figure 6 Bars in an angle. Same exit on the left side.

Figure 4 Bar in an oblique position.
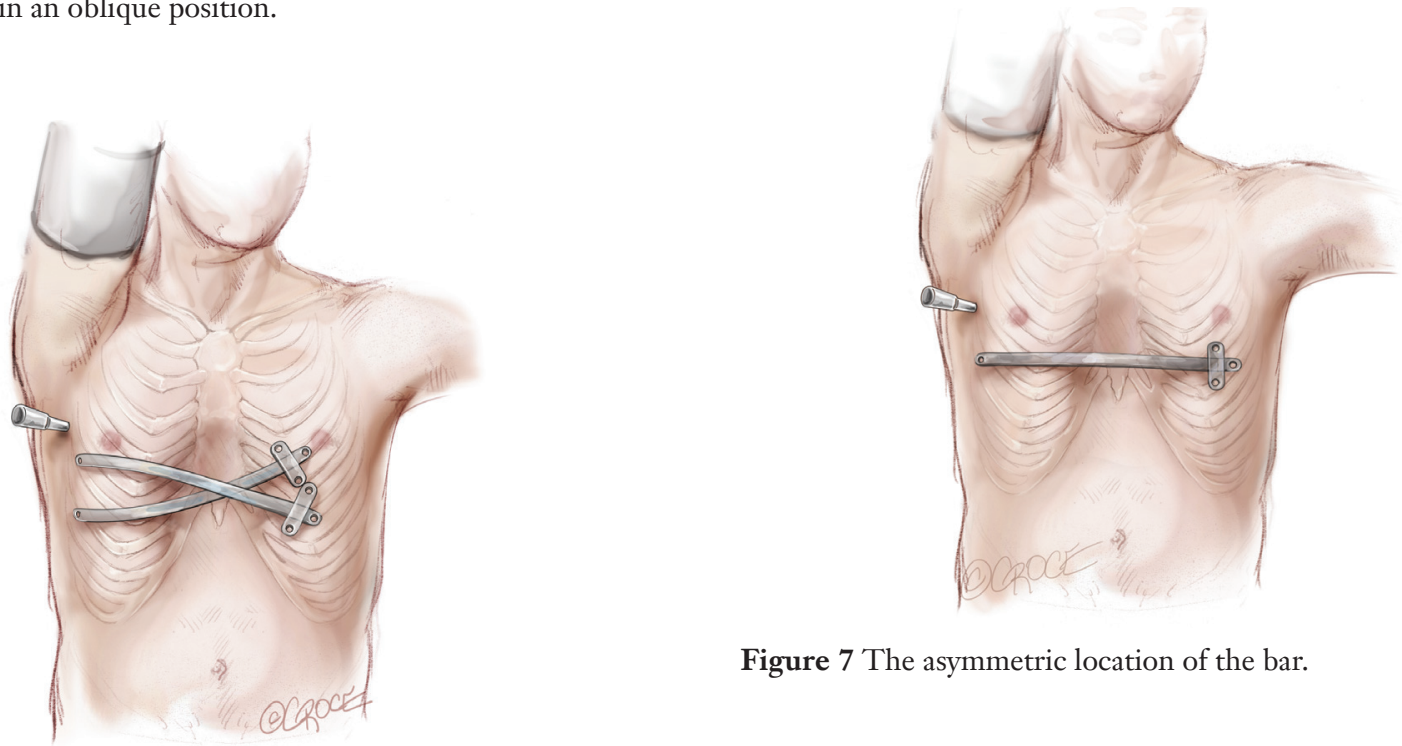

Figure 7 The asymmetric location of the bar.

Figure 5 Crossed bars.

cross or angle configuration (Figures 5,6). One must always thoroughly inspect the chest to see where the support is needed. A template is constructed according to the desired shape of the chest wall. The length of the bar should be such that one end is long enough to cover two ribs and the other end should have just enough space for the stabilizer. This means that the bar is placed asymmetrically on the chest wall (Figure 7). The stabilizer is normally placed on the left side of the patient, which will always have a base of two ribs. The bar is bent in the same way as the template but with some degree of overcorrection due to the pressure of the chest wall, which tends to straighten the bar (Figure 8). The bar is placed on the anterior chest wall and the ends are marked. Small $1-2 \mathrm{~cm}$ incisions are made in the skin. If the location of the bar is at the level of the nipples or higher, the bar should remain beneath the pectoral muscles. In this case, a periareolar incision should be made on the side where the stabilizer will remain. In those cases where two bars are needed with just one rib in between, the bars may be implanted through the same incision.

\section{Introducers}

It is very important that the correct introducer is used 


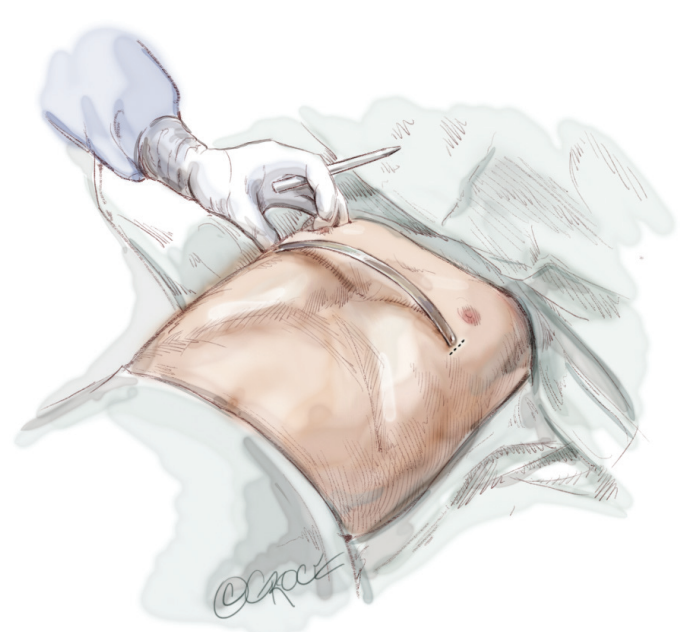

Figure 8 Shows how much the bar should be overcorrected.

to create the tunnel. It is guided under the sternum by the thoracoscope and the tip should be in close contact with the chest wall to avoid damage to the pericardium or heart. In most cases, a large introducer is appropriate; however, in the case of a very deep deformity, an extralarge introducer may be necessary to maintain contact with the chest wall. Due to its long tip, placement of a steel wire in the pinhole to pull facilitates insertion of the introducer through the chest wall (Figure 9). With this technique, stripping of the intercostal muscles is avoided. If the correction needs more than one bar and requires separate incisions, at least two of the introducers should be placed before replacement with the bars to facilitate turning of the bars.

The bar is guided through the pre-formed tunnel by a ligature, which is placed in the pinhole of the introducer before it is retracted. With the introducer in place, the shape of the chest wall should be adjusted by elevating the introducer and pressing on its curvature. If the chest is inflexible, elevation of the introducer for 1-2 minutes optimizes this adjustment, which facilitates rotation of the bar. The bar is inserted in a U-shaped configuration and due to the short distance between the incision and exit from the chest, it is possible to guide the end of the bar manually. The bar is then turned 180 degrees by a rotator. If the excavation is very deep, two rotators may be used to avoid twisting of the bar.

\section{Stabilizer}

One stabilizer is used for every bar to prevent flipping of

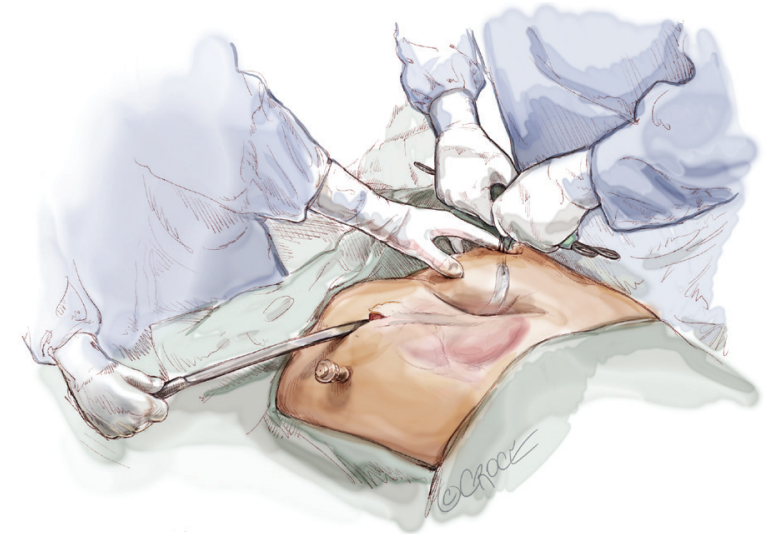

Figure 9 Using a steel wire in the eyelet of the extra large introducer to pull the tip out of the chest before pushing the introducer against the left.

the bar. The stabilizer should be placed close to the incision of the chest wall to optimize fixation of the bar. Stabilizers are placed on the same side. They may cross each other but this does not result in any problems. The stabilizer should be secured to the bar either by a steel wire around the bar end lateral to the stabilizer, or by bending the end with the rotator, thus locking the stabilizer at the bar. If two bars are inserted through the same incision, it is not possible to have two introducers inside at the same time. In this situation, it is appropriate to place one bar first followed by the second one and complete stabilization of the second bar. Following this, the first bar is withdrawn to bend it further before fixation. With the position of the stabilizer close to the hinge point of the bar on the anterior surface of the chest, it is normally not necessary to fixate the end without the stabilizer with additional sutures.

\section{Completion}

The thoracic cavity is exsufflated using a thin chest drain connected to an underwater seal and the drain is subsequently removed. The skin is closed in two layers and sealed by steristrips. The patient is extubated before leaving the operating theatre.

\section{Antibiotics and pain management}

Intravenous antibiotics are administered preoperatively and continued for three days. We use cefuroxime $1,500 \mathrm{mg}$ three times daily and gentamycin $240 \mathrm{mg}$ in the morning. Pain management consists of three to four weeks of 


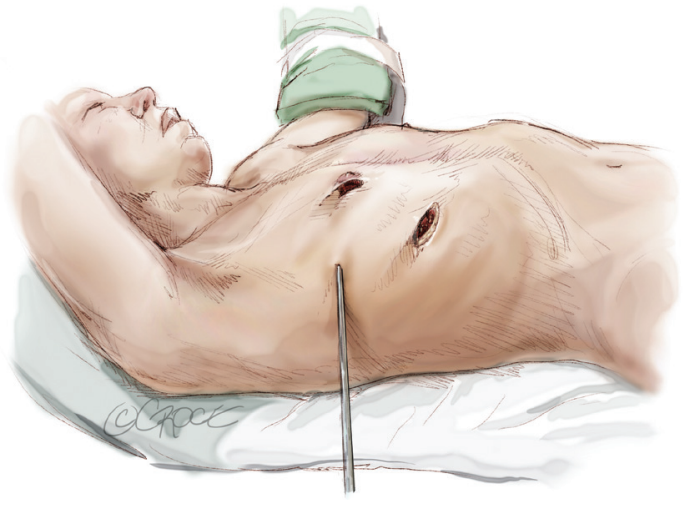

Figure 10 Pectus excavatum after correction.

ibuprofen $400 \mathrm{mg}$ three times daily and four to five weeks of paracetamol 1,000 $\mathrm{mg}$ four times daily, which are started on the day of the surgery. Opioid analgesia is provided for 10 to 14 days, starting on postoperative day one. An initial regimen may consist of oxycodone $10-20 \mathrm{mg}$ twice daily. Epidural analgesia is given as a combination of morphine and bupivacaine on the day of the operation, is changed to pure bupivacaine the following day, and removed on postoperative day two along with the urinary catheter.

\section{Postoperative care and follow-up}

The patient is transferred to the recovery room after surgery for one to two hours and then back to the ward. The patient is encouraged to mobilize the following day. $\mathrm{X}$-ray imaging is carried out on postoperative day two (10) and more than $85 \%$ of patients are discharged from hospital on this second day postoperatively. Six weeks after discharge, the patient is seen in the outpatient clinic for a clinical evaluation and $\mathrm{X}$-ray. No further visits are planned but patients may contact the department. Before surgery, it is important to inform patients what to expect regarding pain. They are encouraged to start physiotherapy four weeks before surgery. Postoperative restrictions to activity include no heavy lifting during the first six weeks postoperatively, with only $2 \mathrm{~kg}$ carried in front of the body and $5 \mathrm{~kg}$ on the shoulders, as well as no cycling. Patients are advised to sleep on their back and minimize twisting movements of the upper body. After six weeks, they may resume normal exercise and and may carry weight in a normal way, In addition, patients are told avoid participating in contact sports for the whole time that they have a bar or bars in situ.

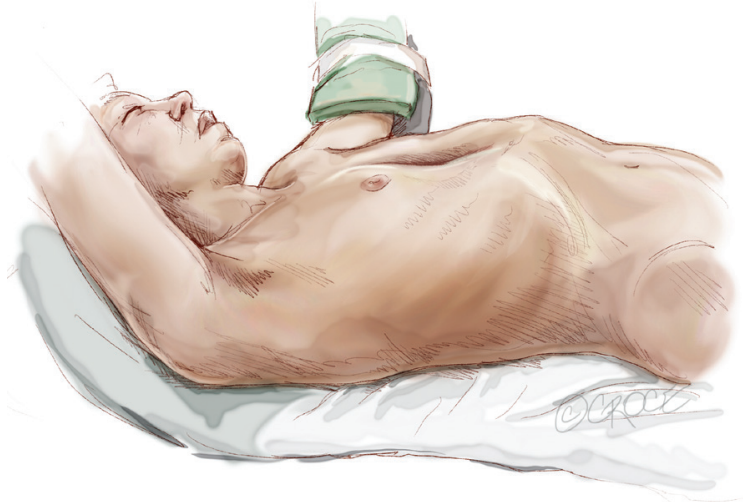

Figure 11 Pectus excavatum before correction.

\section{Clinical results}

The technique with the short bar has a short operation time (median 31 minutes; range, 12-270 minutes) and may result in less pain as $85 \%$ of patients are discharged on the second day postoperatively. Because of the short distance between the hinge point and the stabilizer, the system is very stable and the flipping rate is approximately $1 \%$ (7). The clinical result after correction is shown in Figure 10 compared to before correction in Figure 11.

\section{Advantages}

The short bar technique facilitates insertion of the introducer because of the short distance between the skin incisions and the entrance and exit of the chest wall, which also facilitate bar placement. .In addition, the bar may be guided by finger at the exit point through the chest wall. The position of the stabilizer very close to the hinge point stabilizes the system extremely well, such that the flipping rate is very low and no additional sutures are necessary. The short bar also facilitates bar removal due to its round shape, whereby in most cases it is only necessary to open the skin incision at the site of the stabilizer and then retract the bar with a hook.

\section{Caveats}

Even though the bar should be short, it is important that it is not too short, as this may result in that the end without the stabilizer dropping into the thorax and compressing the heart (11). The end without the stabilizer should cover two ribs to avoid this complication. 


\section{Discussion}

One of the problems with the use of long bars as per the original technique was instability of the bars. Initially, around $15 \%$ of the bars flipped; however, with the addition of a stabilizer, fixation of this stabilizer and additional sutures opposite the stabilizer, the flipping rate was reduced to approximately $1 \%$ (12). This is equivalent to the results with the short bar, which is only held in place by the stabilizer. The short bar is easier to guide through the chest using the surgeon's fingertip, due to the short distance between the incision and the exit point of the bar from the chest cavity. Placing the stabilizer close to the hinge point may also increase the stability of the system. The patient may experience less pain and more than $85 \%$ of patients can be discharged on the second day postoperatively. The short bar is easily removed (13), probably due to its round shape, in contrast to the longer bars with two long bended ends.

\section{Conclusions}

The short bar is very stable and does not require additional sutures or other fixation techniques. It is easier to insert than the longer bar because of the short distance between incisions and entrance and exit points of the chest. Furthermore, it is easier to remove because, in most cases, it is only necessary to open the incision at the site of the stabilizer and then pull the bar out with a hook.

\section{Acknowledgements}

None.

\section{Footnote}

Conflicts of Interest: The author is a consultant at ZimmerBiomet.

\section{References}

1. Maagaard M, Tang M, Ringgaard S, et al. Normalized cardiopulmonary exercise function in patients with pectus excavatum three years after operation. Ann Thorac Surg

Cite this article as: Pilegaard HK. Short Nuss bar procedure. Ann Cardiothorac Surg 2016;5(5):513-518. doi: 10.21037/ acs.2016.09.06
2013;96:272-8.

2. Udholm S, Maagaard M, Pilegaard H, et al. Cardiac function in adults following minimally invasive repair of pectus excavatum. Interact Cardiovasc Thorac Surg 2016;22:525-9.

3. Ravitch MM. The Operative Treatment of Pectus Excavatum. Ann Surg 1949;129:429-44.

4. Nuss D, Kelly RE Jr, Croitoru DP, et al. A 10-year review of a minimally invasive technique for the correction of pectus excavatum. J Pediatr Surg 1998;33:545-52.

5. Pilegaard HK, Licht PB. Early results following the Nuss operation for pectus excavatum--a single-institution experience of 383 patients. Interact Cardiovasc Thorac Surg 2008;7:54-7.

6. Pilegaard HK, Licht PB. Can absorbable stabilizers be used routinely in the Nuss procedure? Eur J Cardiothorac Surg 2009;35:561-4.

7. Pilegaard HK. Nuss technique in pectus excavatum: a mono-institutional experience. J Thorac Dis 2015;7:S172-6.

8. Jaroszewski DE, Ewais MM, Chao CJ, et al. Success of Minimally Invasive Pectus Excavatum Procedures (Modified Nuss) in Adult Patients ( $\geq 30$ Years). Ann Thorac Surg 2016;102:993-1003.

9. Kelly RE Jr. Pectus excavatum: historical background, clinical picture, preoperative evaluation and criteria for operation. Semin Pediatr Surg 2008;17:181-93.

10. Knudsen MR, Nyboe C, Hjortdal VE, et al. Routine postoperative chest $\mathrm{X}$-ray is unnecessary following the Nuss procedure for pectus excavatum. Interact Cardiovasc Thorac Surg 2013;16:830-3.

11. Maagaard M, Udholm S, Hjortdal VE, et al. Right ventricular outflow tract obstruction caused by a displaced pectus bar 30 months following the Nuss procedure. Eur J Cardiothorac Surg 2015;47:e42-3.

12. Nuss D. Recent experiences with minimally invasive pectus excavatum repair "Nuss procedure". Jpn J Thorac Cardiovasc Surg 2005;53:338-44.

13. Nyboe C, Knudsen MR, Pilegaard HK. Elective pectus bar removal following Nuss procedure for pectus excavatum: a single-institution experience. Eur J Cardiothorac Surg 2011;39:1040-2. 